\title{
A STOCHASTIC REPRESENTATION OF SUBGRID UNCERTAINTY FOR DYNAMICAL CORE DEVELOPMENT
}

\author{
Aneesh Subramanian, Stephan Juricke, Peter Dueben, and Tim Palmer
}

\begin{abstract}
A new paradigm for dynamical core development is presented where the precision of dynamical cores should not be more than the level of uncertainty provided by the stochastic scheme presented in this study.
\end{abstract}

0 ne of the great challenges since the advent of computational science has been the realistic simulation and prediction of weather and climate. Numerical models developed for simulating the weather and climate are characterized by a

AFFILIATIONS: SUBRAMANIAN-University of Colorado Boulder, Boulder, Colorado, and Department of Physics, University of Oxford, Oxford, United Kingdom, and Scripps Institution of Oceanography, University of California, San Diego, La Jolla, California; JURICKe,* Dueben,* ANd PALmer-Department of Physics, University of Oxford, Oxford, United Kingdom

* CURRENT AFFILIATIONS: JURICKE-Jacobs University Bremen, Bremen, and Alfred Wegener Institute, Helmholtz Centre for Polar and Marine Research, Bremerhaven, Germany; DUEBENECMWF, Reading, United Kingdom

CORRESPONDING AUTHOR: Aneesh Subramanian,

aneeshcs@colorado.edu

The abstract for this article can be found in this issue, following the table of contents.

DOI:10.II75/BAMS-D-17-0040.I

In final form 21 December 2018

(C)2019 American Meteorological Society

For information regarding reuse of this content and general copyright

information, consult the AMS Copyright Policy. dynamical core that solves the dynamical NavierStokes equations or some approximations thereof, and physical parameterizations to represent subgridscale processes. The dynamical core describes the truncation of the underlying differential equations onto a finite representation in the form of grid points, finite-element basis functions, or spectral basis functions, and the corresponding algorithms that evolve the resolved scales of motion. Complementary to this, physical parameterizations are traditionally deterministic formulas that provide estimates of the gridscale effect of processes (e.g., deep convection) that cannot be resolved by the dynamical core.

Global atmospheric models have been using subgrid-scale parameterizations for more than four decades to represent the wide range of physical processes (e.g., convection, radiation, gravity wave drag) that occur on horizontal scales smaller than the grid spacing (Arakawa and $\mathrm{Wu}$ 2013; Plant and Yano 2015). Cloud and convection parameterizations have proven to be long-term challenges because of the many processes and many scales involved. Several approaches have been developed to represent atmospheric convection in highly simplified parameterization schemes in global climate models (GCMs) over 
the past five decades (Smith 2013; Plant and Yano 2015). More recently, as individual components of weather forecasting models continue to be improved and run at resolutions where convection is permitted or resolved, increased consideration is given to the coupling of physics and dynamics in these models (Gross et al. 2016, 2018).

Dynamical cores are developed and compared in various different flavors and are used on many different types of grids [such as Gaussian grids, yin-yang, cubed-sphere, and others as described in Jablonowski and Williamson (2006), Lauritzen et al. (2010), and Ullrich et al. (2017)]. While dynamical cores with different discretization methods and different grids will certainly have very different properties, it is difficult to compare the solutions of dynamical cores beyond their performance in simple test cases that are based on linear behavior [e.g., the Williamson test suite in Williamson (2007)]. To fairly compare the performance of dynamical cores in strongly nonlinear regimes, we need ensemble simulations that evolve the flow-dependent uncertainty. A large number of papers discuss ways to improve accuracy (see, e.g., Lauritzen et al. 2011, and references therein), for example, by the removal of spurious modes for linear waves or grid imprinting (e.g., for the edges of the cubed sphere). Yet, it is difficult to judge how much these features will actually impact the dynamics of an atmosphere or ocean model at full complexity (Williamson 2007; Jablonowski and Williamson 2011).

The error growth in predictive models of our atmosphere is related to errors in our model formulation. The larger the model error, the more severe the error growth and the sooner we lose forecast skill. This destructive interaction between initial time and model errors is a consequence of chaos in such predictions, as was noted by the mathematician Henri Poincaré in his book Science and Hypothesis (Poincaré 1905) and then developed by Lorenz in a series of landmark scientific papers (e.g., Lorenz 1969).

In recent years, the representation of model uncertainty in climate model predictions has become an important addendum to the development of climate models. Different techniques have been developed, the most common approach being multimodel ensembles, for example, as described in the Fourth Assessment Report of the Intergovernmental Panel on Climate Change (Trenberth et al. 2007), and perturbed parameter ensembles (e.g., Stainforth et al. 2005). However, a third technique has emerged: the stochastic-dynamic parameterization where the treatment of tendencies due to unresolved processes is by stochastic terms rather than bulk-formula deterministic representations (Lorenz 1975; Pitcher 1977; Palmer 2001).

Stochastic parameterization is the approach to represent uncertainty in the model formulation with stochastic terms. A more detailed motivation for including stochastic parameterizations in our current weather and climate models is presented in Palmer (2012). Successful applications of stochastic approaches in numerical weather prediction (NWP) have evolved in the recent decade, from the early attempts in the European Centre for Medium-Range Weather Forecasts (ECMWF) ensemble prediction system (Palmer et al. 1993) to operational schemes at several meteorological services around the world to generate ensemble forecasts from short-range to seasonal time scales (Teixeira and Reynolds 2008; Doblas-Reyes et al. 2009; Berner et al. 2009; Stockdale et al. 2011; Palmer et al. 2008, 2009b; Palmer 2012; Sušelj et al. 2013, 2014; Sanchez et al. 2016).

The currently operational medium-range ensemble prediction system at ECMWF uses the stochastically perturbed physical tendencies (SPPT; Buizza et al. 1999; Palmer et al. 2009a) scheme as one method to represent model uncertainty. The SPPT scheme applies stochastic perturbations in the form of multiplicative noise to the parameterized diabatic part of the tendency equations of the prognostic variables (Palmer et al. 2009a; Weisheimer et al. 2014; Berner et al. 2015). SPPT has been shown to improve the quality of NWP forecasts, and also to nonlinearly rectify some of the biases in the climate mean and variability in the ECMWF model (Weisheimer and Palmer 2014; Weisheimer et al. 2014). Recent studies have also shown other positive impacts of the SPPT scheme on other areas, such as on model representation of tropical rainfall variability in atmosphere-only simulations in three different global models (Watson et al. 2017), on Madden-Julian oscillation (MJO) propagation in atmosphere-only integrations with the EC-EARTH model (Davini et al. 2017), and on the El Niño-Southern Oscillation in coupled climate simulations with the Community Climate System Model, version 4 (Christensen et al. 2017).

The effect of uncertainties due to the finite truncation of the model can be estimated by running ensembles of integrations, whose members represent independent realizations of a stochastic process that describes these truncation uncertainties. The numerical model solutions are uncertain beyond just the numerical errors from the numerical schemes used to discretize the equations. A large contribution to the uncertainty in model integrations comes 
from the uncertainty in the tendencies of large-scale convection and other key physical processes in the atmosphere. A schematic of a mesoscale convective system in the midlatitudes interacting with a midlatitude dynamical wave is shown in Fig. 1 . This symbolizes that the errors in physical parameterizations translate to errors in dynamics in the dynamical core. Hence, accounting for these errors is necessary to have a robust forecasting system that captures the uncertainty in forecasts.

The model uncertainty described above can be approximated using an additive stochastic noise with an amplitude equivalent to the quantified uncertainty in the solutions. This technique is especially attractive as it is agnostic to the numerical schemes used or other parameterizations developed in the model. It is simpler to implement than inherently solving stochastic differential equations for the system. Hence, it is an approach that can be used for intercomparison of different models with an adequate uncertainty representation. We propose such an additive noise test case for the dynamical core development effort.

Importantly, tendencies of momentum and other state variables associated with subgrid-scale processes in the dynamical core are uncertain and hence need to be represented as a stochastic process. Our approach is to represent the uncertainty in the subgrid-scale processes with an additive noise term in the dynamical tendencies for a Held-Suarez test case. This can be further extended to other stochastic test cases where uncertainty in model dynamics and physics can be tested simultaneously. We discuss how using this additive noise term in the dynamical core for testing and development can help develop future models for probabilistic predictions.

We can represent the state of the system (atmosphere in our case) at a time $t$ by a variable $x_{t}$, which is a three-dimensional field of several variables. The model $F$ integrates $x_{t}$ forward in time. The external forces including subgrid-scale forcing can be represented as a part of $F$. The time stepping in this model is represented as $x_{t+1}=F\left(x_{t}, y_{t}\right)$. In this case, $y_{t}$ is an error in our estimate of $x_{t}$ due to errors in physical processes (e.g., diffusion, momentum mixing, convective momentum transport) that contribute to the tendency of $x_{t}$. The inherent inaccuracy of the deterministically parameterized subgrid-scale processes is largely independent of the detailed parameterization schemes themselves.

We propose to represent this inherent inaccuracy in weather and climate models using a stochastic additive term derived from the ECMWF operational forecast model. The goal of this study is to provide an

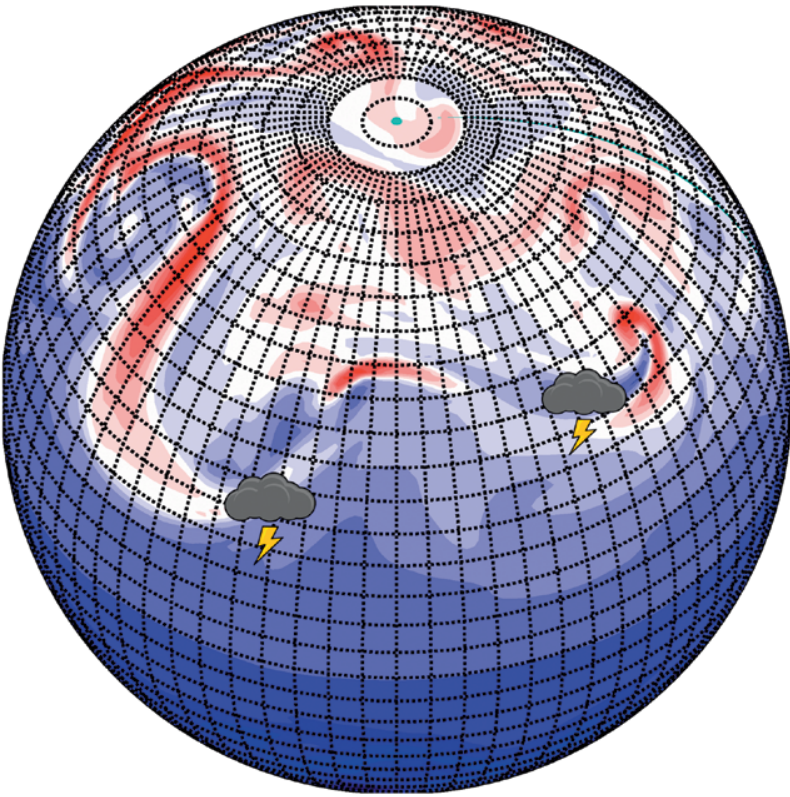

Fig. I. Schematic of thunderstorms and large convective cells embedded in a numerical grid for a dynamical core. The color contours show the upper-atmosphere potential vorticity field for one time step of the IFS dynamical core Held-Suarez run. The interaction of convective clouds with the large-scale wave indicates instabilities and error propagations that can arise from these fundamental physical processes in the atmosphere.

estimate of uncertainty in dynamical cores and set a benchmark for dynamical cores to compare against. We will provide a low-cost stochastic scheme that can be "bolted" onto a dynamical core and would (as best we can judge it) represent the inherent inaccuracy of the subgrid parameterizations as a function of model resolution. The essential corollary is that there would be no point trying to develop dynamical cores that are more precise than the levels of noise that the stochastic schemes induce. There are naturally other important properties that also need to be taken into account when considering the quality of a dynamical core, such as conservation properties and the accurate representation of balanced flows (Staniforth and Thuburn 2012). Yet, we argue that these properties of the dynamical core should be considered along with the aspects of computational cost and what we gain or lose in terms of forecast skill or model fidelity. We will present results for a sample benchmark test case and encourage other dynamical core developers to engage in an intercomparison project for a stochastic test case of all dynamical cores.

The paper is organized as follows. The second section describes the derivation of the stochastic term. The third section describes the implementation of the 
stochastic test case. The fourth section summarizes the results from the experiments with stochastic noise added to the dynamical core. Discussions about the framework of testing and developing dynamical cores with a stochastic approach, and including stochastic additive terms to represent model error, are presented in the last section.

\section{MODEL AND DERIVATION OF THE} ADDITIVE STOCHASTIC FORCING.

ECMWF's Integrated Forecasting System (IFS) handles climate variability and biases well, especially in the extratropical regions (Weisheimer and Palmer 2014). It also has a sophisticated treatment of model-generated uncertainties. Many of the physical processes in the IFS atmospheric model are parameterized, for example, those related to atmospheric convection and clouds, radiative processes in the atmosphere, and boundary layer turbulence. In the IFS model, these unresolved diabatic processes are described through a set of deterministic physical parameterization schemes.

The SPPT (Palmer et al. 2009a) scheme is one of the stochastic parameterization schemes included in the IFS to represent uncertainty in its physical parameterization tendencies for moisture, heat, and momentum. An early implementation of this scheme is described in Buizza et al. (1999). A detailed description of its implementation in the operational ECMWF model is presented for the first time in Palmer et al. (2009a), while a more recent description of the operational version of SPPT is described in Leutbecher et al. (2017). The current operational implementation of the scheme is a three-scale perturbation scheme. In the SPPT scheme, the sum of the physics tendencies of the prognostic variables (e.g., temperature, humidity, and momentum) at each time step are perturbed by a stochastic multiplicative term. The scheme has predefined spatiotemporal decorrelation scales that generate smooth perturbation patterns. The implementation makes use of the spectral pattern generator present in IFS to define these perturbation coefficients. A univariate distribution is chosen for the perturbations that is independent of the variables to be perturbed. The scheme follows

$$
\mathbf{X}_{p}=(1+r \mu) \mathbf{X},
$$

where $\mathbf{X}_{p}$ is the perturbed tendency vector for all prognostic variables, that is, horizontal velocities $(u, v)$, potential temperature $T$, and humidity $q$; $\mathbf{X}=(\partial / \partial t)(u, v, T, q)$ are the parameterized tendencies from the deterministic schemes; and $r$ is the random perturbation pattern. Parameter $\mu \in[0,1]$ is the factor used for reducing the perturbation amplitude close to the surface and in the stratosphere, as perturbations in the near-surface regions have undesirable effects which lead to instabilities in the solution (Leutbecher et al. 2017), while perturbations in the stratosphere are not desired due to the negligible uncertainty in the clear sky radiation tendencies in the stratosphere.

The three spatiotemporal decorrelation scales of $r$ follow an autoregressive model of order 1 (AR1). The characteristics of the three scales of perturbations are: i) $500-\mathrm{km}$ and $6-\mathrm{h}$ decorrelation representing mesoscale uncertainty; ii) 1,000-km and 3-day decorrelation representing the synoptic-scale uncertainties in physical tendencies; and iii) 2,000-km and 30-day decorrelation representing the uncertainty in the large-scale impact of subgrid-scale physics tendencies. A linear combination of these three scales is used to account for model errors at different temporal and spatial scales. The variance of each of these stochastic terms is predefined and represents the magnitude of the uncertainty in each of these scales. The shortest spatial scale is defined to have the largest variance signifying that the largest uncertainty lies in the mesoscale physical tendencies.

Coarse-graining studies with cloud-resolving models (Palmer et al. 2009a; Shutts and Palmer 2007) as well as reanalysis tendency increments have motivated the choices for these perturbation patterns. Shutts and Palmer (2007) showed that the standard deviation of the temperature tendency in a high-resolution cloudresolving simulation is linearly related to the mean temperature tendency. This state dependence of the standard deviation to the mean tendency supports the use of a multiplicative noise scheme like SPPT for modeling uncertainty. Some theories such as Craig and Cohen (2006) argue that errors in temperature tendency terms derived by convective parameterization behave like a Poisson process. This is also shown in numerical results from a more recent coarsegraining study by Shutts and Pallarès (2014), where they examined the relationship between the width of the probability distribution and the mean value of the tendency of the coarser-resolution model. They find different relationships for different physical processes parameterized in the model. Hence, these recent findings imply that there is scope for further development of SPPT. In the following section we describe benchmark test case setup that uses the SPPT tendencies for stochastic forcing in the dynamical cores.

\section{APPLYING THE STOCHASTIC FORCING IN BENCHMARK TESTS. We run a simulation} of IFS in an aquaplanet setup (Malardel and Wedi 
2016) with the stochastic physics switched on and save the dynamical tendencies from the SPPT scheme at every time step for zonal and meridional winds. The dynamical tendencies from the SPPT scheme provide an estimate of uncertainty in the dynamics that derives from the physical parameterized processes in the system. Figure 2 shows values of the SPPT dynamical tendencies as a function of latitude and longitude for two different vertical levels (850 and $500 \mathrm{hPa}$, in the left panels). The right panels show the SPPT dynamical tendencies at two different latitudes (equator and $10^{\circ} \mathrm{N}$ ) as a function of pressure levels and longitude. This is an estimate of uncertainty that quantifies an error bound which defines the level of precision required in dynamical cores. We then use these terms as additive noise for a dry Held-Suarez test case in an idealized experiment with simple physics and a spectral dynamical core. The model is run forward for 1,500 days with additive stochastic noise at every time step. The model time step is $30 \mathrm{~min}$ in the semiLagrangian dynamical core. The test case without stochasticity is the conventional Held-Suarez test case where the dynamical core equations are integrated until equilibrium with no additional uncertainty represented.

We want to provide a test bed that allows us to compare the error from the dynamics and physics in the dynamical core in comparison to the subgrid-scale noise that can be expected at the given
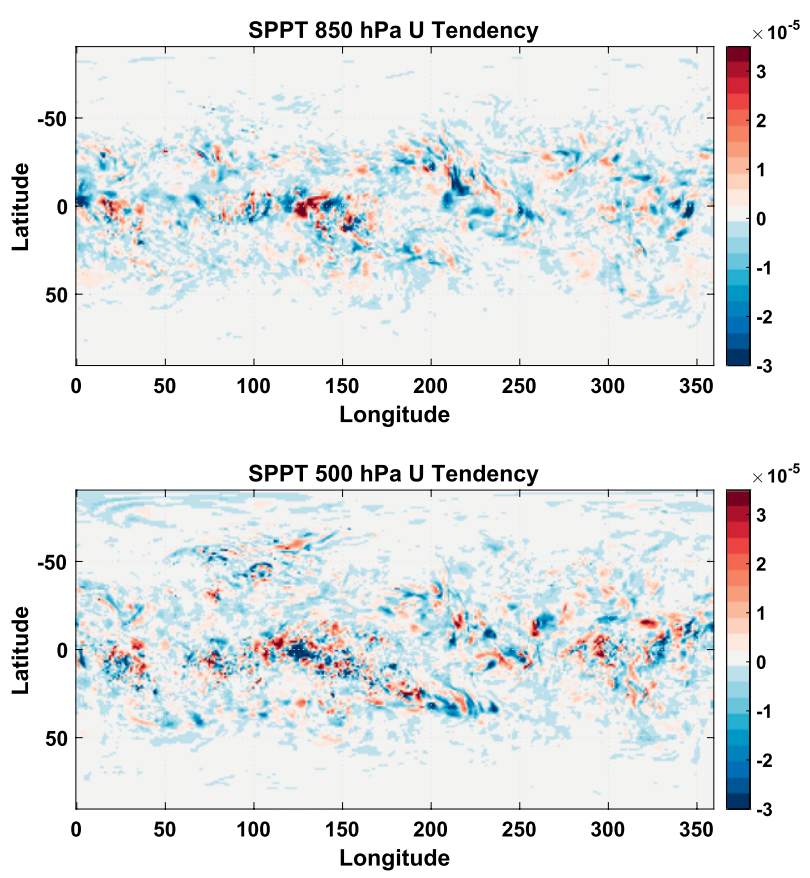

resolution. Hence, these saved SPPT tendencies serve as the quantified uncertainty in the subgrid-scale processes and can be used for such a test case. We first implement and test this in the IFS model dynamical core. If feasible and of interest to the dynamical core developer groups, we have a portable version of the quantified uncertainty values which can, in principle, be implemented in the different dynamical cores. A link to a website where the dataset for the noise term and scripts to set up the test case is given in the "Discussion and conclusions" section.

Deterministic Held-Suarez test case. The Held-Suarez test case uses a dry atmosphere, an ideal gas on a spherical rotating planet forced only by radiative fluxes modeled as a simple relaxation of temperature to a prescribed radiative equilibrium. This relaxation is a function of latitude and pressure and a frictional force that relaxes the flow near the surface to zero (in the reference frame rotating with the surface). The model equations are described in Held and Suarez (1994).

The test case is designed to capture some of the complexity of midlatitude jets and storm tracks on a rotating sphere. The climate that emerges (the statistics of the winds and temperatures) shares several features of Earth's climate. Figure 3 shows the vertical temperature profile of the model mean climate as a zonal-mean time-mean temperature state for
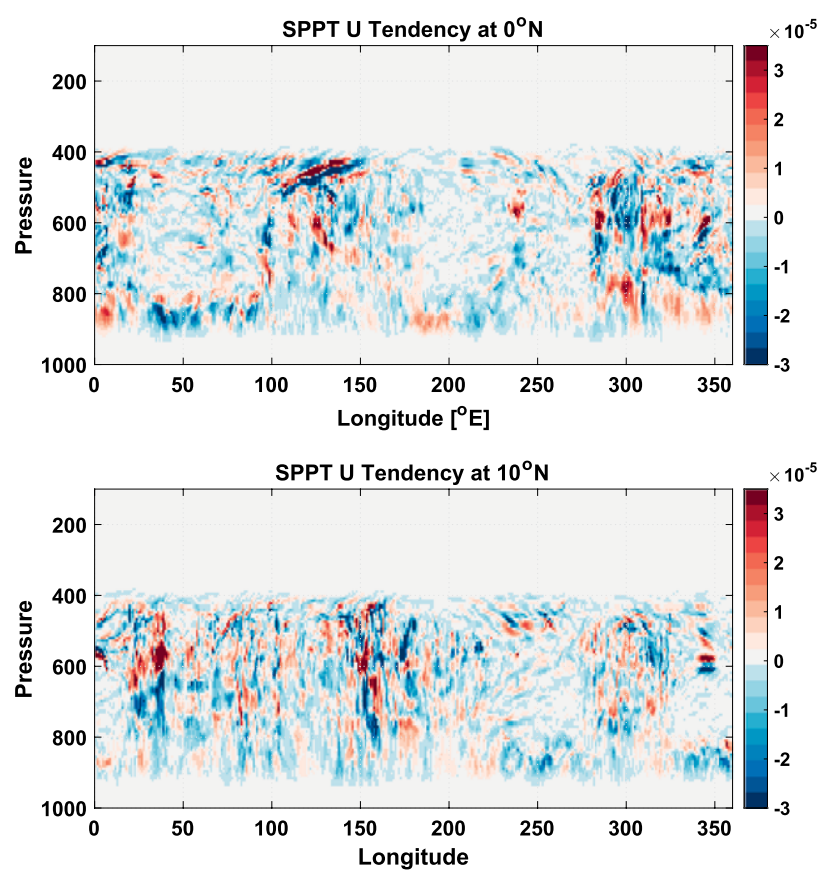

FIG. 2. SPPT dynamical tendencies $\left(\mathrm{m} \mathrm{s}^{-2}\right)$ from one time step in IFS. (left) SPPT dynamical tendencies as a function of latitude and longitude at (top) 850 and (bottom) $500 \mathrm{hPa}$. (right) SPPT dynamical tendencies at (top) the equator and (bottom) $10^{\circ} \mathrm{N}$ as a function of pressure levels (1,000-100 hPa) and longitude. 

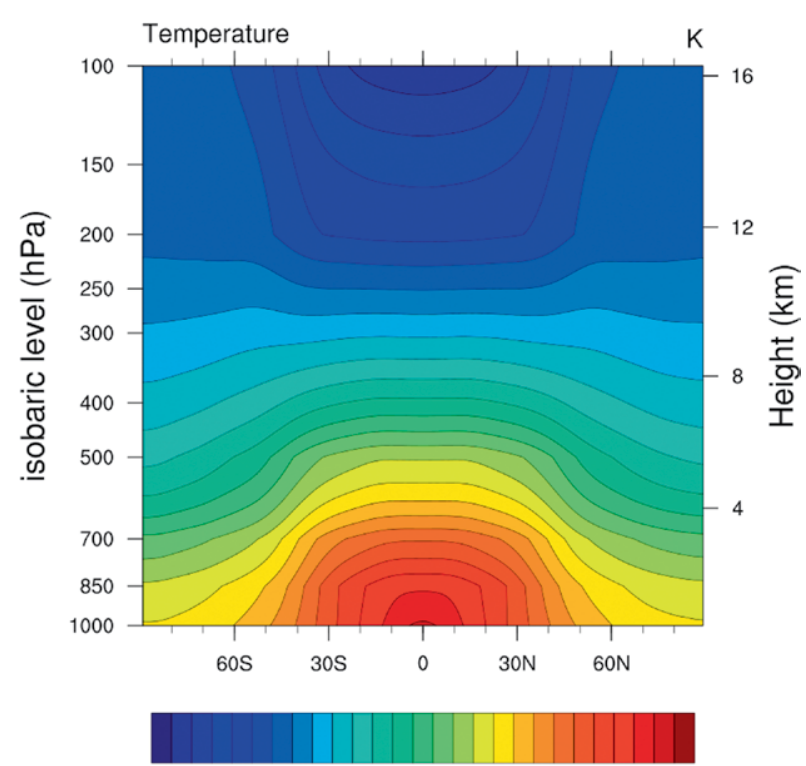

$185200215230 \quad 245 \quad 260 \quad 275 \quad 290 \quad 305$

Fig. 3. Zonal-mean temperature profile averaged over the last 1,000 -day period in a 1,500 -day simulation of the deterministic Held-Suarez test case.

the test case. The prescribed radiative equilibrium specifies a positive static stability in the tropics and this decreases as we move poleward in order to reduce gravity wave-driven instabilities in the tropics. For the deterministic run, we use the IFS spectral model which is a standard hydrostatic, $\sigma$-coordinate, semi-Lagrangian, semi-implicit, spectral transform model. The model is used with 137 vertical levels and a T159 horizontal resolution, meaning that all fields are expressed as sums over the spherical harmonics with a total of 159 wavenumbers (this translates into a grid spacing of approximately $125 \mathrm{~km}$ ). The temperature profile globally is relaxed to a radiative-convective equilibrium profile. Figure 4 shows the time-averaged zonally averaged zonal winds (east-west) produced by this test case. The contour interval is $2 \mathrm{~m} \mathrm{~s}^{-1}$. The zero contour meets the surface near $30^{\circ}$ and $60^{\circ}$ latitude.

The basic idea of the Held-Suarez test case is to validate dynamical cores by evaluating the long-term statistical properties of a balanced three-dimensional global circulation. The test case does not include such external forcings as varying boundary condition at seasonal or even longer time scales. Relaxation of temperature to the prescribed radiative equilibrium takes effect on a time scale of 40 days. The $e$-folding time for the Rayleigh friction is 1 day at Earth's surface. The statistics calculated over 1,000 days, as proposed in Held and Suarez (1994), are good representatives of the basic features of the simulated climate state.
Stochastic Held-Suarez test case. We have designed a stochastic forcing dataset that can be used as an additive noise term for dynamical cores when performing benchmark test cases. Here we use the Held-Suarez test case with forcing and dissipation. We start with an atmospheric state over a rotating spherical surface that is in equilibrium. There is no topography at the surface and hence the surface is at a constant geopotential as in an aquaplanet simulation. The only specified dissipation in the system is a simple linear damping of the velocities in the spectral model equations. The temperatures are relaxed to a prescribed "radiative equilibrium" $T_{\mathrm{eq}}$, which is a function of the latitude and height.

The benchmark test case developed here for dynamical core comparisons in the presence of uncertainty in the solutions is defined as a stochastic Held-Suarez test case. We perform long-term integrations with the IFS dynamical core with and without additive stochastic noise from the SPPT tendencies. The test case with the additive stochastic noise has the three-dimensional stochastic term added at every grid point.

We use the spectral IFS model described in the previous section. The model is run for two identical twin experiments. One is the deterministic test case with no additive noise term in the dynamical equations. The second experiment is the stochastic test case where the zonal and meridional momentum

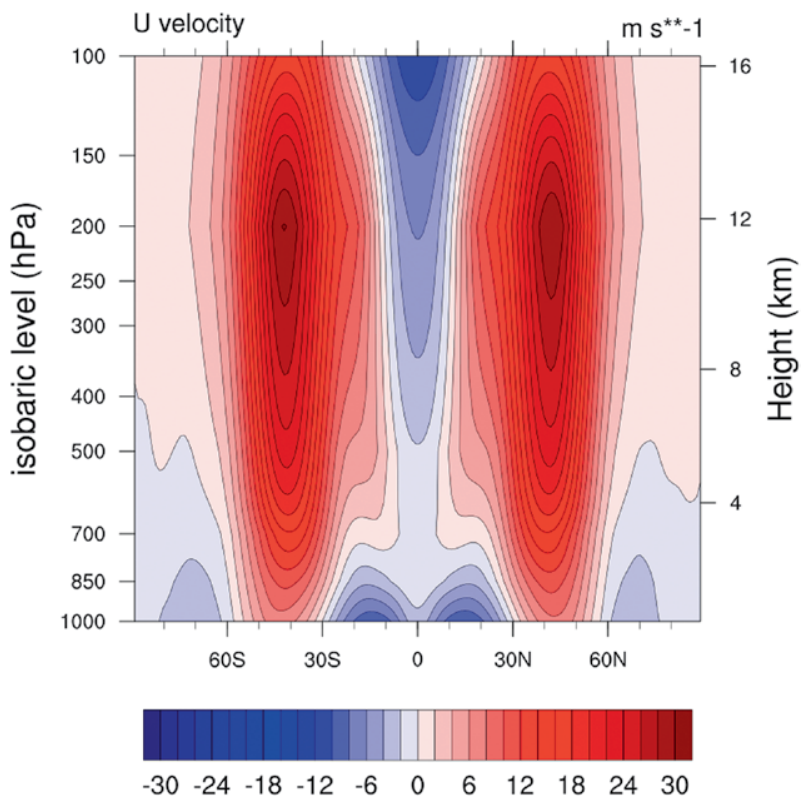

Fıg. 4. Zonal-mean wind profile as a function of vertical pressure levels and latitude. The field is time-averaged over the last I,000-day period in a 1,500-day simulation for the deterministic Held-Suarez run. This is an average over the 1,000 days following a 500-day spinup. 
equations have an additional noise term which is randomly drawn from a sample of the SPPT dynamical tendencies. The two model runs cover a time period of 1,500 days. The last 1,000-day sequence taken during this time period is used to compute the time-mean values which are good representatives of the model's climate. In addition to the time average, the data are zonally averaged. This extracts the meridional (north-south)-oriented model circulation.

RESULTS. The time-mean zonal-mean zonal wind patterns highlight the jet streams in the midlatitudes with the maximum westerly wind speeds (Fig. 4). The surface and upper-level winds in the equatorial region are easterlies.

The differences between the deterministic zonalmean zonal wind fields (left panel in Fig. 5) and the same field from the stochastic integration of the model (the right panel in Fig. 5) are on the order of 5\% or less, indicating that the additive noise term, though representing uncertainty in the system, does not change the mean state solution considerably. Hence, using this estimate of uncertainty in the model is appropriate as it does represent the uncertainty in the dynamics of the system arising from inaccurate physics parameterizations in the operational models but does not lead to a large mean-state change.

Figure 6 shows the meridional eddy heat flux $\left[v^{\prime} T^{\prime}\right]$, which is one of the important energy transport mechanisms that establish the global circulation in the model. This covariance term indicates that heat is transported from the equatorial heat surplus regions to the poles where the heat budget becomes negative. As can be seen in the figure, the eddy heat transports in both experiments are similar with mostly small differences (less than 5\%) in the solutions. Figure 7 shows the kinetic energy spectra for $500-\mathrm{hPa}$ winds (multiplied by the cube of the wavenumber for ease of interpretation) for the two experiments showing a similar slope for both the stochastic and deterministic simulations.

This range of differences in the two solutions indicates the uncertainty range in solutions that
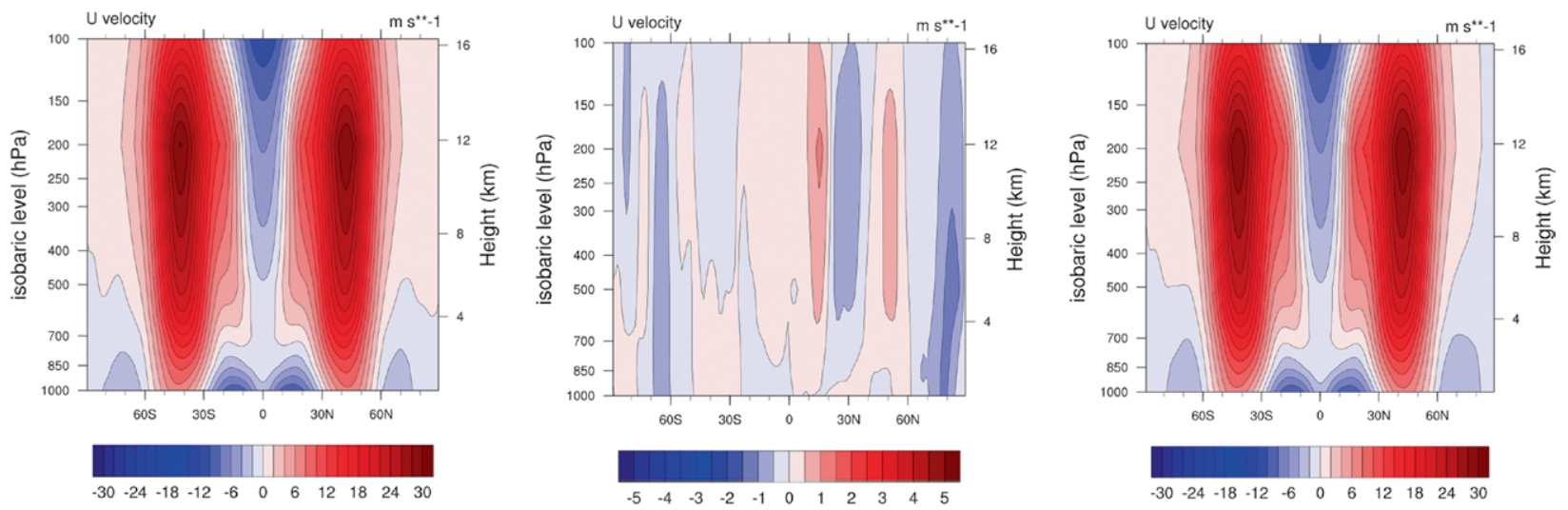

FIG. 5. Mean zonal wind profile for (left) deterministic and (right) stochastic Held-Suarez runs and (center) a difference map as a function of height. The fields are averaged for the last I,000 days of a I,500-day simulation.
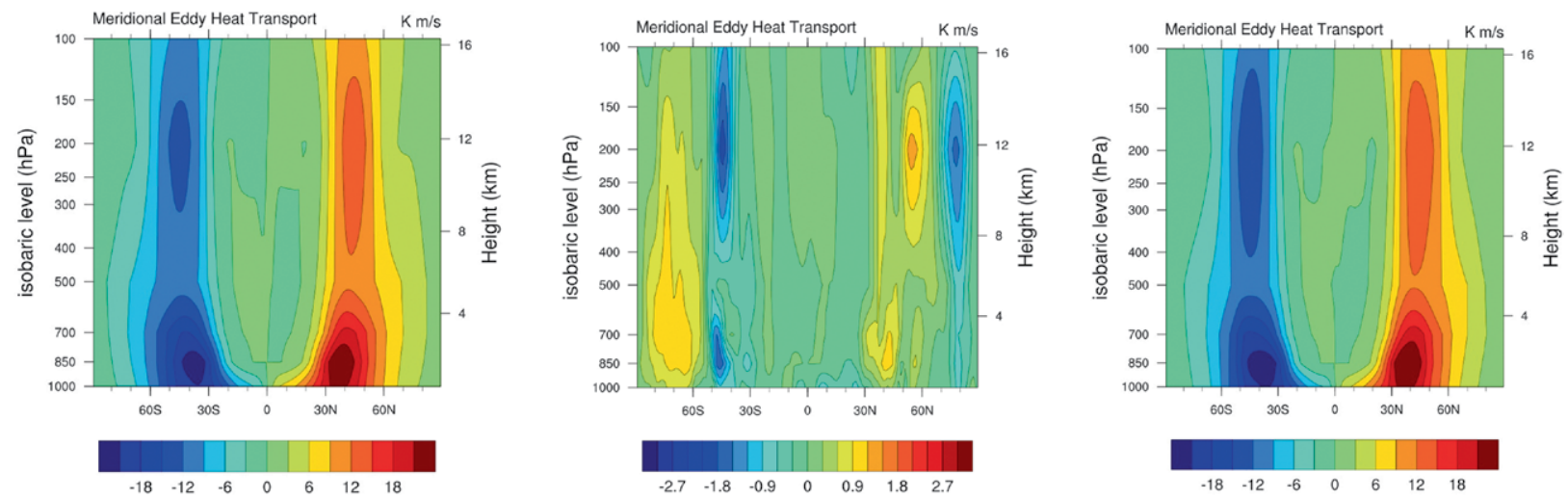

Fig. 6. Meridional eddy heat transport $\left[v^{\prime} T^{\prime}\right]$ profile for (left) deterministic and (right) stochastic Held-Suarez runs and (center) a difference map as a function of height. The fields are averaged for the last 1,000 days of a I,500-day simulation. 
dynamical core developers should be aware of when developing accurate dynamical cores for operational weather and climate models. Model solutions differing by amplitudes greater than the differences indicated in results above imply a significant difference in accuracy and should be considered when comparing two dynamical cores. The solution of two different dynamical cores should be considered identical if the difference in their solutions is not greater than the difference in the above-stated stochastic dynamical core test case. Their evaluation should then consider other aspects such as scalability and computational efficiency.

These test results show the amplitude of error in model solutions when the additive stochastic noise term is active. The mean climate and statistical characteristics of the additive stochastic Held-Suarez test case compared to the deterministic test case represent the uncertainty in the physical parameterizations present in GCMs. These statistical differences quantify the uncertainty in the dynamical cores given the uncertainty in current-generation model physics since SPPT is a quantified stochastic term in a stateof-the-art weather and climate model (Leutbecher et al. 2017). We can now use this uncertainty in dynamical core test cases to compare and contrast

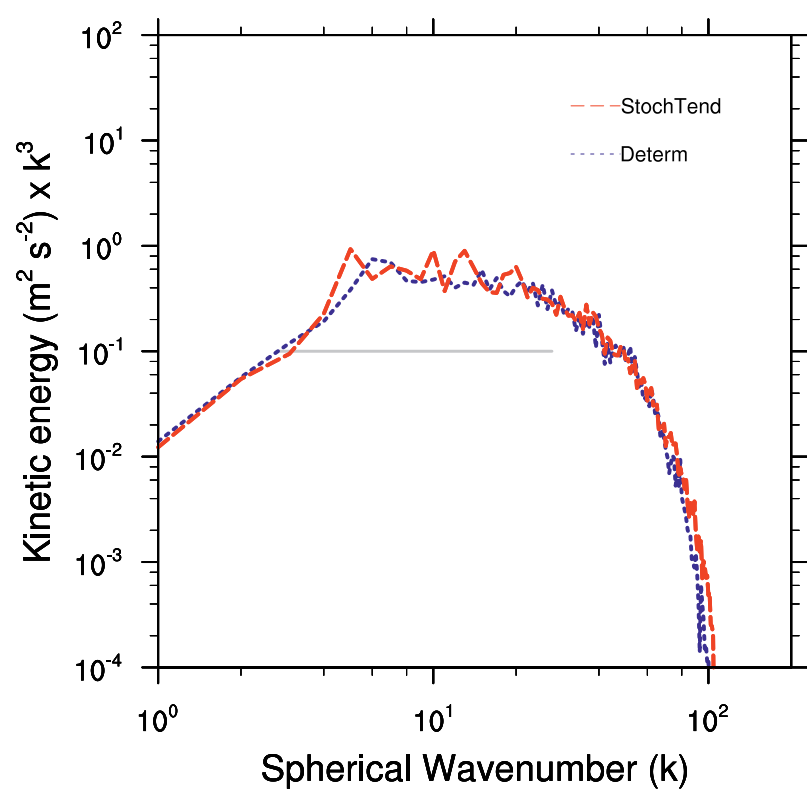

FIG. 7. Kinetic energy spectra for the deterministic (blue) and stochastically perturbed tendency (red) Held-Suarez test case runs for the $500-\mathrm{hPa}$ pressure level in the atmosphere. Kinetic energy is averaged over the last I,000 days of the simulation. The gray line shows the spectral slope for $k^{-1}$, where $k$ is the wavenumber. For clarity, the spectra have been multiplied by $\boldsymbol{k}^{3}$. dynamical core solutions, especially in the context of numerical accuracy and computational costs.

Intercomparison of different dynamical cores with this stochastic test case can be done with an ensemble set of integrations. The ensemble spread in these integrations, especially in the tropics, would very likely be greater with the additive noise [as seen in ensemble numerical weather prediction, e.g., Fig. 2b in Palmer et al. (2009a)]. This would imply that if we were looking to test the impact of some change in the numerical schemes, the change would have to be stronger in the model with noise than in the model without noise, in order for the impact to be deemed different at the 5\% level. Ongoing research in developing a scale-selective reduced precision IFS model shows that the statistical significance of the impact of precision on model results does depend on whether the models are run with or without SPPT (Chantry et al. 2018).

\section{DISCUSSION AND CONCLUSIONS.}

Representing model uncertainty with stochastic parameterizations has become common in weather and climate simulations. Hence, a revision of the deterministic numerical schemes should be undertaken to ensure the convergence of the numerical solutions for stochastic models. The development of more than a dozen dynamical cores by modeling centers around the globe necessitates intercomparison and evaluation of these dynamical cores and their merits and demerits, especially in the context of stochastic parameterization.

We are interested in developing stochastic test cases or benchmark standards for these dynamical core intercomparisons of atmospheric climate models. The stochastic test case should satisfy two conditions to be useful: define an uncertainty bound on the solutions of the dynamical core and represent the long-term climate and statistical properties of the realistic global circulations.

Reducing the computational cost of weather and climate simulations would lower energy consumption per simulation. From the standpoint of reducing costs, the use of reduced precision arithmetic has become an active area of research. Research groups study the impact of using single-precision arithmetic on simulation accuracy with different dynamical cores (Düben and Palmer 2014; Düben et al. 2015; Váňa et al. 2017; Nakano et al. 2018).

An example of an application of the stochastic dynamical core test case is to implement the additive noise term in a dynamical core that is integrated in double precision. The solutions from a deterministic double-precision dynamical core test case can be 
compared to a dynamical core that is integrated at single precision and if the difference is smaller than that between the double-precision dynamical core and a double-precision stochastic dynamical core, we then state that the single-precision dynamical core solution is within the uncertainty bound of the physics in the system. Hence, the single-precision dynamical core results are classified as equivalent to the double-precision dynamical core. We can further test other reduced precision configurations of dynamical cores to evaluate what precisions would increase the errors in the solutions beyond those in the stochastic dynamical core test case. This precision could then serve as a benchmark precision for further development of the dynamical core.

It makes little sense in trying to develop dynamical cores that are more precise than the level of uncertainty in the solutions achievable by weather and climate models. We quantify this uncertainty using a version of the ECMWF IFS weather forecasting model using the stochastic physics scheme in this model and running an aquaplanet simulation. The velocity tendencies from the stochastic scheme are saved and used as an additive noise term in the stochastic benchmark test case.

We describe a low-cost stochastic scheme, which can be integrated into any existing dynamical core at the development stage. The stochastic terms are made available publicly, and help and guidance can be provided for designing experiments for the intercomparison of dynamical cores in the presence of uncertainty. A dataset for the additive stochastic term can be downloaded (https://sites.google.com /site/aneeshcs/research/stochastic_dycore). There is also a description of the test case and sample code for preparing the additive stochastic term for different dynamical cores.

We hope that other groups will join in this effort of comparing dynamical cores with an added stochastic term such that the solutions are evaluated with error bounds. If the different dynamical cores are sufficiently similar with the added stochastic term, the precision of the numerics and computational costs of the dynamical cores should be compared to evaluate which ones are more beneficial to run given the limits on computation.

We present a fundamentally new paradigm to adjust the accuracy of deterministic dynamical cores. Overall, this will ensure that future climate models are computationally efficient, and hence, we can expend the saved computation cost on higher-resolution models and increased ensemble size to account for uncertainty in our solutions.
The prototype test case introduced in this paper can be extended further to develop better stochastic schemes that are in tune with the model dynamical core. We will be able to generate estimates of uncertainty in the dynamics using higher-resolution dynamical cores and SPPT stochastic physics schemes in the future that can be applied to different-resolution model test cases. We look forward to a discussion on how these stochastic test cases for dynamical cores can be applied and extended in the model development community.

ACKNOWLEDGMENTS. We thank the four anonymous reviewers whose comments and reviews helped improve this manuscript. We thank Glenn Carver, Filip Vana, Antje Weisheimer, Judith Berner, Sylvie Malardel, and Christiane Jablonowski for helpful discussions. We thank the ERC (Grant 291406) for supporting ACS, PD and SJ. We would also like to specially thank the OpenIFS project for making this work possible.

\section{REFERENCES}

Arakawa, A., and C. Wu, 2013: A unified representation of deep moist convection in numerical modeling of the atmosphere. Part I. J. Atmos. Sci., 70, 1977-1992, https://doi.org/10.1175/JAS-D-12-0330.1.

Berner, J., G. J. Shutts, M. Leutbecher, and T. N. Palmer, 2009: A spectral stochastic kinetic energy backscatter scheme and its impact on flow-dependent predictability in the ECMWF Ensemble Prediction System. J. Atmos. Sci., 66, 603-626, https://doi .org/10.1175/2008JAS2677.1.

— , K. R. Smith, S. Y. Ha, J. P. Hacker, and C. Snyder, 2015: Increasing the skill of probabilistic forecasts: Model-error representations versus calibration and debiasing. Mon. Wea. Rev., 143, 1295-1320, https:// doi.org/10.1175/MWR-D-14-00091.1.

Buizza, R., M. Milleer, and T. N. Palmer, 1999: Stochastic representation of model uncertainties in the ECMWF ensemble prediction system. Quart. J. Roy. Meteor. Soc., 125, 2887-2908, https://doi.org/10.1002/qj .49712556006 .

Chantry, M., T. Thornes, T. N. Palmer, and P. Düben, 2018: Scale-selective precision for weather and climate forecasting. Mon. Wea. Rev., 147, 645-655, https://doi.org/10.1175/MWR-D-18-0308.1.

Christensen, H., J. Berner, D. R. B. Coleman, and T. N. Palmer, 2017: Stochastic parameterization and El Niño-Southern Oscillation. J. Climate, 30, 17-38, https://doi.org/10.1175/JCLI-D-16-0122.1.

Craig, G. C., and B. G. Cohen, 2006: Fluctuations in an equilibrium convective ensemble. Part I: Theoretical 
formulation. J. Atmos. Sci., 63, 1996-2004, https:// doi.org/10.1175/JAS3709.1.

Davini, P., and Coauthors, 2017: Climate SPHINX: Evaluating the impact of resolution and stochastic physics parameterisations in the EC-Earth global climate model. Geosci. Model Dev., 10, 1383-1402, https://doi.org/10.5194/gmd-10-1383-2017.

Doblas-Reyes, F. J., and Coauthors, 2009: Addressing model uncertainty in seasonal and annual dynamical ensemble forecasts. Quart. J. Royal Meteor. Soc., 135, 1538-1559, https://doi.org/10.1002/qj.464.

Düben, P., and T. Palmer, 2014: Benchmark tests for numerical weather forecasts on inexact hardware. Mon. Wea. Rev., 142, 3809-3829, https://doi.org/10.1175 /MWR-D-14-00110.1.

—, F. Russell, X. Niu, W. Luk, and T. Palmer, 2015: On the use of programmable hardware and reduced numerical precision in earth-system modeling. $J$. Adv. Model. Earth Syst., 7, 1393-1408, https://doi .org/10.1002/2015MS000494.

Gross, M., S. Malardel, C. Jablonowski, and N. Wood, 2016: Bridging the (knowledge) gap between physics and dynamics. Bull. Amer. Meteor. Soc., 97, 137-142, https://doi.org/10.1175/BAMS-D-15-00103.1.

— , and Coauthors, 2018: Physics-dynamics coupling in weather, climate, and earth system models: Challenges and recent progress. Mon. Wea. Rev., 146, 3505-3544, https://doi.org/10.1175/MWR -D-17-0345.1.

Held, I. M., and M. J. Suarez, 1994: A proposal for the intercomparison of the dynamical cores of atmospheric general circulation models. Bull. Amer. Meteor. Soc., 75, 1825-1830, https://doi.org/10.1175/1520 -0477(1994)075<1825:APFTIO>2.0.CO;2.

Jablonowski, C., and D. L. Williamson, 2006: A baroclinic instability test case for atmospheric model dynamical cores. Quart. J. Roy. Meteor. Soc., 132, 2943-2975, https://doi.org/10.1256/qj.06.12.

—, and — 2011: The pros and cons of diffusion, filters and fixers in atmospheric general circulation models. Numerical Techniques for Global Atmospheric Models, Springer, 381-493.

Lauritzen, P. H., C. Jablonowski, M. A. Taylor, and R. D. Nair, 2010: Rotated versions of the Jablonowski steady-state and baroclinic wave test cases: A dynamical core intercomparison. J. Adv. Model. Earth Syst., 2, 15, https://doi.org/10.3894/JAMES.2010.2.15.

,,$-- \ldots$, and _-, Eds., 2011: Numerical Techniques for Global Atmospheric Models. Lecture Notes in Computational Science and Engineering, Vol. 80, Springer, $564 \mathrm{pp}$.

Leutbecher, M., and Coauthors, 2017: Stochastic representations of model uncertainties at ECMWF: State of the art and future vision. Quart. J. Roy. Meteor. Soc., 143, 2315-2339, https://doi.org/10.1002/qj.3094.

Lorenz, E. N., 1969: The predictability of a flow which possesses many scales of motion. Tellus, 21, 289-307, https://doi.org/10.3402/tellusa.v21i3.10086.

—, 1975: Climatic predictability. The physical basis of climate and climate modelling: Report of the International Study Conference in Stockholm, GARP Publ. Series 16, WMO, 132-136, https://library.wmo .int/pmb_ged/garp-ps_16.pdf.

Malardel, S., and N. P. Wedi, 2016: How does subgrid-scale parametrization influence nonlinear spectral energy fluxes in global NWP models? J. Geophys. Res. Atmos., 121, 5395-5410, https://doi .org/10.1002/2015JD023970.

Nakano, M., H. Yashiro, C. Kodama, and H. Tomita, 2018: Single precision in the dynamical core of a nonhydrostatic global atmospheric model: Evaluation using a baroclinic wave test case. Mon. Wea. Rev., 146, 409-416, https://doi.org/10.1175/MWR-D-17-0257.1.

Palmer, T. N., 2001: A nonlinear dynamical perspective on model error: A proposal for non-local stochasticdynamic parametrization in weather and climate prediction models. Quart. J. Roy. Meteor. Soc., 127, 279-304, https://doi.org/10.1002/qj.49712757202.

—, 2012: Towards the probabilistic Earth-system simulator: A vision for the future of climate and weather prediction. Quart. J. Roy. Meteor. Soc., 138, 841-861, https://doi.org/10.1002/qj.1923.

-, F. Molteni, R. Mureau, R. Buizza, P. Chapelet, and J. Tribbia, 1993: Ensemble prediction. Proc. ECMWF Seminar on Validation of Models over Europe, Vol. 1, Reading, United Kingdom, European Centre for Medium-Range Weather Forecasts, 21-66.

—, F. J. Doblas-Reyes, A. Weisheimer, and M. J. Rodwell, 2008: Toward seamless prediction: Calibration of climate change projections using seasonal forecasts. Bull. Amer. Meteor. Soc., 89, 459-470, https://doi.org/10.1175/BAMS-89-4-459.

—, R. Buizza, F. Doblas-Reyes, T. Jung, M. Leutbecher, G. Shutts, M. Steinheimer, and A. Weisheimer, 2009a: Stochastic parametrization and model uncertainty. ECMWF Tech. Memo. 598, 42 pp., https://doi .org/10.21957/ps8gbwbdv.

—, F. J. Doblas-Reyes, A. Weisheimer, and M. J. Rodwell, 2009b: Reply. Bull. Amer. Meteor. Soc., 90, 1551-1554, https://doi.org/10.1175/2009BAMS2916.1.

Pitcher, E. J., 1977: Application of stochastic dynamic prediction to real data. J. Atmos. Sci., 34, 3-21, https:// doi.org/10.1175/1520-0469(1977)034<0003:AOSDPT $>2.0 . \mathrm{CO} ; 2$.

Plant, R. S., and J.-I. Yano, Eds., 2015: Parameterization of Atmospheric Convection, World Scientific, $1172 \mathrm{pp}$. 
Poincaré, H., 1905: Science and Hypothesis. Science Press, $244 \mathrm{pp}$.

Sanchez, C., K. D. Williams, and M. Collins, 2016: Improved stochastic physics schemes for global weather and climate models. Quart. J. Roy. Meteor. Soc., 142, 147-159, https://doi.org/10.1002/qj.2640.

Shutts, G. J., and T. N. Palmer, 2007: Convective forcing fluctuations in a cloud-resolving model: Relevance to the stochastic parameterization problem. J. Climate, 20, 187-202, https://doi.org/10.1175/JCLI3954.1.

— tion uncertainty associated with horizontal resolution in numerical weather prediction models. Philos. Trans. Roy. Soc. London, 372A, 20130284, https://doi. org/10.1098/rsta.2013.0284.

Smith, R. K., Ed., 2013: The Physics and Parameterization of Moist Atmospheric Convection. Nato Science Series, Vol. 505, Springer, 498 pp.

Stainforth, D. A., and Coauthors, 2005: Uncertainty in predictions of the climate response to rising levels of greenhouse gases. Nature, 433, 403-406, https://doi .org/10.1038/nature03301.

Staniforth, A., and J. Thuburn, 2012: Horizontal grids for global weather and climate prediction models: A review. Quart. J. Roy. Meteor. Soc., 138, 1-26, https:// doi.org/10.1002/qj.958.

Stockdale, T. N., and Coauthors, 2011: ECMWF seasonal forecast system 3 and its prediction of sea surface temperature. Climate Dyn., 37, 455-471, https://doi .org/10.1007/s00382-010-0947-3.

Sušelj, K., J. Teixeira, and D. Chung, 2013: A unified model for moist convective boundary layers based on a stochastic eddy-diffusivity/mass-flux parameterization. J. Atmos. Sci., 70, 1929-1953, https://doi .org/10.1175/JAS-D-12-0106.1.

_ , T. F. Hogan, and J. Teixeira, 2014: Implementation of a stochastic eddy-diffusivity/mass-flux parameterization into the Navy Global Environmental Model. Wea. Forecasting, 29, 1374-1390, https://doi .org/10.1175/WAF-D-14-00043.1.
Teixeira, J., and C. A. Reynolds, 2008: Stochastic nature of physical parameterizations in ensemble prediction: A stochastic convection approach. Mon. Wea. Rev., 136, 483-496, https://doi.org/10.1175 /2007MWR1870.1.

Trenberth, K. E., and Coauthors, 2007: Observations: Surface and atmospheric climate change. Climate Change 2007: The Physical Science Basis, S. Solomon et al., Eds., Cambridge University Press, 235-336.

Ullrich, P. A., and Coauthors, 2017: DCMIP2016: A review of non-hydrostatic dynamical core design and intercomparison of participating models. Geosci. Model Dev., 10, 4477-4509, https://doi.org/10.5194 /gmd-10-4477-2017.

Váňa, F., P. Düben, S. Lang, T. Palmer, M. Leutbecher, D. Salmond, and G. Carver, 2017: Single precision in weather forecasting models: An evaluation with the IFS. Mon. Wea. Rev., 145, 495-502, https://doi .org/10.1175/MWR-D-16-0228.1.

Watson, P. A. G., J. Berner, S. Corti, P. Davini, J. von Hardenberg, C. Sanchez, A. Weisheimer, and T. N. Palmer, 2017: The impact of stochastic physics on tropical rainfall variability in global climate models on daily to weekly time scales. $J$. Geophys. Res. Atmos., 122, 5738-5762, https://doi .org/10.1002/2016JD026386.

Weisheimer, A., and T. N. Palmer, 2014: On the reliability of seasonal climate forecasts. J. Roy. Soc. Interface, 11, 20131162, https://doi.org/10.1098/rsif.2013 .1162 .

_ , S. Corti, and T. Palmer, 2014: Addressing model error through atmospheric stochastic physical parametrizations: Impact on the coupled ECMWF seasonal forecasting system. Philos. Trans. Roy. Soc. London, 372A, 20130290, https://doi.org/10.1098 /rsta.2013.0290.

Williamson, D. L., 2007: The evolution of dynamical cores for global atmospheric models. J. Meteor. Soc. Japan, 85, 241-269, https://doi.org/10.2151 /jmsj.85B.241. 


\section{Attention AMS Student Members}

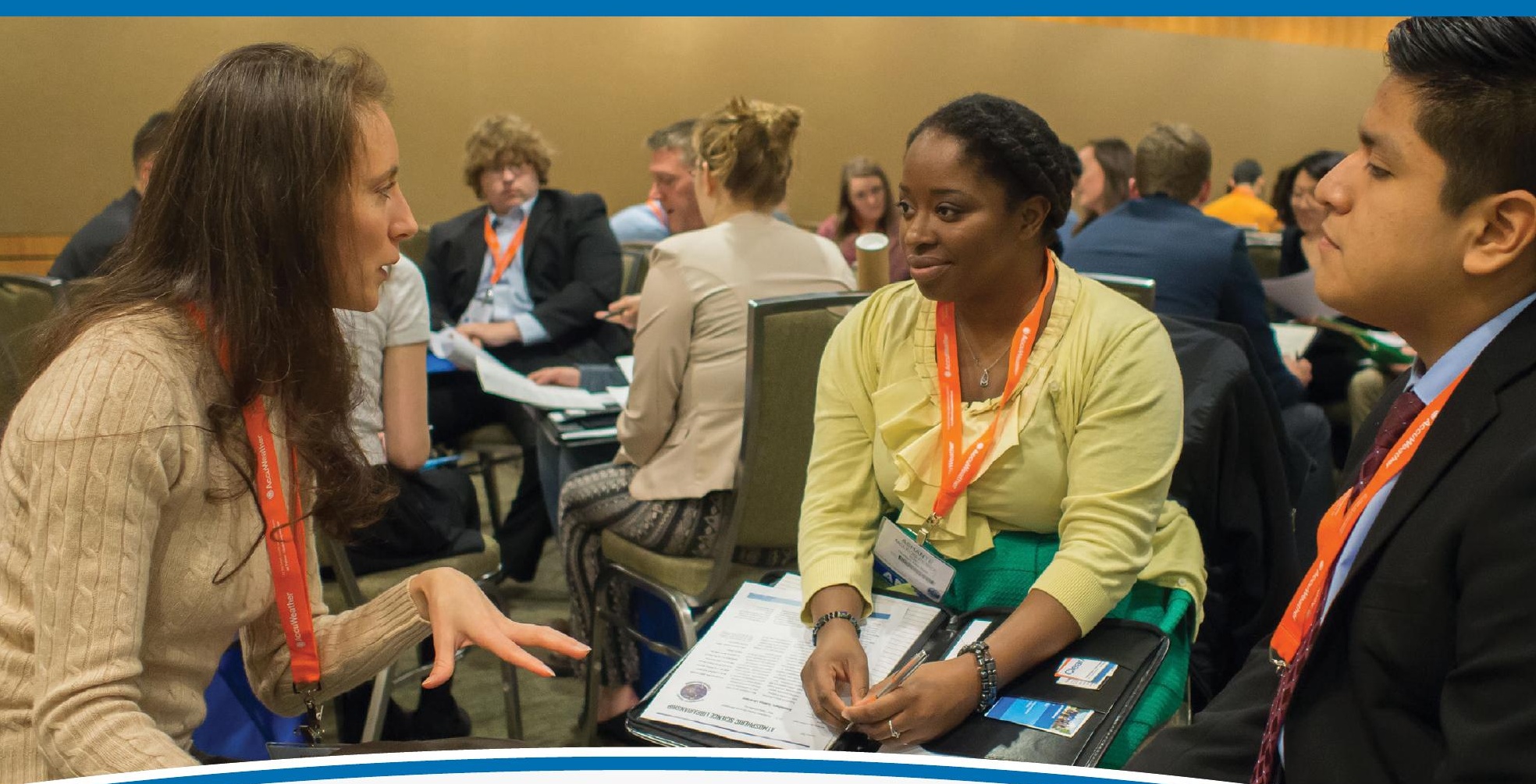

Stay connected to AMS after graduation for half the regular membership rate

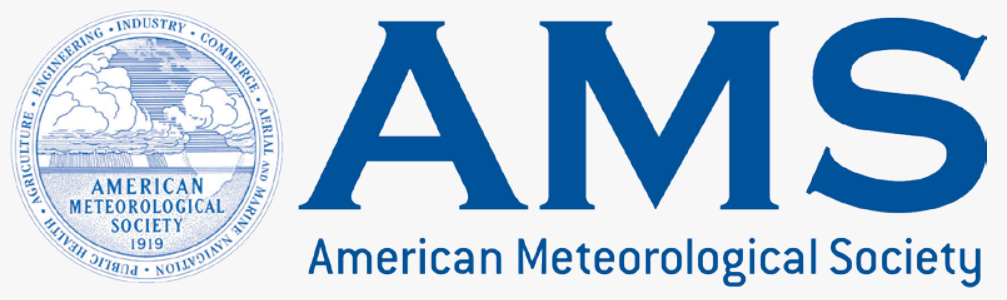

Let AMS help you build your expertise, your network, your career. There's never been a more important time to be a member. 